\title{
An introduction to economic evaluation in occupational therapy: cost-effectiveness of pre-discharge home visits after stroke (HOVIS)
}

\author{
Christopher James Sampson* Marilyn James \\ Phillip Whitehead Avril Drummond
}

May 20, 2015

\section{Publication type:}

Peer-reviewed journal article

\section{This version:}

This is a post-print version of the manuscript, meaning that it has been peerreviewed but may still contain errors.

\section{Published version:}

Sampson, C., James, M., Whitehead, P., \& Drummond, A. (2014). An introduction to economic evaluation in occupational therapy: cost-effectiveness of pre-discharge home visits after stroke (HOVIS). British Journal of Occupational Therapy 77(7), 330-335. DOI: 10.4276/030802214X14044755581664

\section{Copyright:}

This work is licensed under the Creative Commons Attribution 3.0 Unported License. To view a copy of this license, visit http://creativecommons.org/licenses/by/3.0/ or send a letter to Creative Commons, 444 Castro Street, Suite 900, Mountain View, California, 94041, USA.

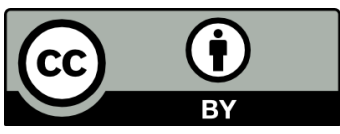

*Correspondence to: C J Sampson, Division of Rehabilitation and Ageing, University of Nottingham, Queen's Medical Centre, Nottingham, NG7 2UH, UK chris.sampson@nottingham.ac.uk 


\title{
An introduction to economic evaluation in occupational therapy: cost-effectiveness of pre-discharge home visits after stroke (HOVIS)
}

\author{
21st January 2014
}

Key words: costs; home assessment; stroke rehabilitation; health economics Word count: 3,100 


\section{Abstract}

\section{Introduction}

Occupational therapy interventions, such as home visits, have been identified as being resource-intensive, but cost-effectiveness analyses are rarely, if ever, carried out. We sought to estimate the cost-effectiveness of occupational therapy home visits after stroke, as part of a feasibility study, and to demonstrate the value and methods of economic evaluation.

\section{Method}

We completed a cost-effectiveness analysis of pre-discharge occupational therapy home visits after stroke compared with a hospital-based interview, carried out alongside a feasibility randomised controlled trial. Our primary outcome was quality-adjusted life years. Full cost and outcome data were available for 65 trial participants.

\section{Results}

We found that the mean total cost of a home visit was $£ 183$, compared with a cost of $£ 75$ for a hospital interview. Home visits are shown to be slightly more effective, resulting in a cost per quality-adjusted life year of just over $£ 20,000$.

\section{Conclusion}

Our analysis is the only economic evaluation of this intervention to date. Home visits are shown to be more expensive and more effective than a hospital-based interview, but our results are subject to a high level of uncertainty and should be treated as such. Further economic evaluations in this field are encouraged. 


\section{Introduction}

The cost-effectiveness of occupational therapy interventions has been identified as a priority area for future research by The College of Occupational Therapists (2007). Information on cost-effectiveness is vital in order to demonstrate that occupational therapy interventions represent value for money for service providers and the public. Healthcare commissioners and practitioners are increasingly required to justify the provision of interventions with constrained budgets in mind. To simply demonstrate the efficacy of an intervention is no longer sufficient in a health service with competing and increasing demands for limited resources. Economic evaluation involves the comparative analysis of alternative courses of action, in terms of both their costs and consequences (Drummond et al., 2005). It provides a means of combining information about the costs and consequences of interventions, within a well-established analysis framework, in order to inform decision-makers about the cost-effectiveness of alternative programs.

One aspect of occupational therapy practice that is perceived to be particularly resource-intensive is the pre-discharge home visit. Home visits are common practice in acute hospital settings in the United Kingdom (Patterson et al., 2001, Welch and Lowes, 2005) and elsewhere (Lannin et al., 2011). These visits are believed to facilitate a safe discharge from hospital (Johnston et al., 2010) and to increase people's ability to cope at home and in the wider community (Lannin et al., 2007). Hibberd (2008) calculated the cost of a home visit and reported this as $£ 135$ per visit, though this calculation was based on only three visits for inpatients of an intermediate care unit. Although occupational therapy home visits, for various patient groups, have been reported to be a costly intervention there is a paucity of information on the actual costs or the cost-effectiveness of these visits (Barras, 2005, Lannin et al., 2007, Patterson and Mulley, 1999). A recent review by Logan et al. (2011) identified no published evidence on the cost-effectiveness of occupational therapy home visits after stroke.

A recent survey of stroke units in England reported that pre-discharge home visits take an average of just under 4 hours of occupational therapy time to complete including travel, organisation and writing-up (Drummond et al., 2012). An Australian survey reported that visits took a mean time of 2 hours including travel time, but did not include time spent organising and writing-up (Lannin et al., 2011). While these figures demonstrate that home visits are a particularly time-intensive intervention, it is not clear what these visits actually cost, what resources are utilised, or whether they are a cost-effective use of occupational therapists' time.

The National Institute for Health and Clinical Excellence (NICE) have published guidelines for the appropriate methods of economic evaluation (National Institute for Health and Clinical Excellence, 2008). Economic evaluations can be carried out alongside randomised controlled trials or can combine existing information from a number of sources to provide estimates. The Home Visits after Stroke (HOVIS) study (Drummond et al., 2013) comprised a feasibility randomised controlled trial and a cohort study, and included an economic evaluation 
estimating the cost-effectiveness of home visits compared with a hospital-based interview. This paper reports on the HOVIS economic evaluation. We introduce some key concepts in economic evaluation, which are necessary to understand the implications of economic results and to conduct future studies of this nature.

\section{Method}

\section{Trial}

A feasibility randomised controlled trial of occupational therapy pre-discharge home visits for patients with a stroke was completed, and has been reported elsewhere (Drummond et al., 2013). Approval was given by the Research Ethics Committee (Berkshire Research Ethics Committee ref 10/H0505/41). All patients were approached for informed, written consent and were free to withdraw from the trial at any stage. Data were included in the analyses up to the point of withdrawal. Drummond et al. (2013) primarily describe the feasibility of the trial, but also report various outcome measures and intervention costs. The treatment group received a pre-discharge home assessment visit with an occupational therapist. The control group received a pre-discharge home assessment structured interview with an occupational therapist in hospital. It is important to note that hospital-based interviews are not currently standard practice, and that current practice varies (Drummond et al., 2012). However, the use of this control intervention was necessary to ensure acceptability of the trial (Drummond et al., 2013). The trial also included a non-randomised cohort group, for whom clinicians believed a home visit was essential. Ninety-three individuals were randomised in the trial; 47 to treatment and 46 to control. Thirty three individuals were recruited to the cohort group, and received a home visit. Baseline assessments were carried out prior to randomisation. Follow-up was completed one month after discharge from hospital. All analyses were carried out on the basis of intention to treat. While the trial collected data from 93 participants, we include only those with available cost and outcomes data in the economic evaluation. This reduced the sample size for the cost-effectiveness analysis to 65; 28 in the control group and 37 in the intervention arm.

\section{Economic evaluation}

The most common form of economic evaluation in healthcare is cost-effectiveness analysis, and we focus on this framework here. Cost-effectiveness analysis is most commonly used in the evaluation of new interventions, in order to assess whether current practice should be replaced. However, as in the case of home visits after stroke, current practice itself can be in place without appropriate evaluation. Economic evaluation can be used to inform the more formal provision (or termination) of an existing intervention, or to highlight uncertainties in current understanding. It is common for more effective interventions to be more expensive. In this case we need to know the additional, or 'incremental', 
cost associated with the extra benefit. Using this information, decision-makers can then decide whether the incremental benefit justifies the incremental cost.

It is worthwhile considering some alternatives to cost-effectiveness analysis. A more basic form of evaluation is cost-minimisation analysis, in which the costs of two (or more) interventions are compared but clinical outcomes are demonstrably equivalent in each group. However, it is rarely the case that alternative interventions are clinically equivalent and it is usually more appropriate to formally combine information about the costs and consequences of alternative approaches. Cost-benefit analysis is a common form of economic evaluation (Drummond et al., 2005). It involves the quantification of all costs and consequences in monetary terms, allowing for direct quantification of tradeoffs between the two. However, this method can be problematic in healthcare due to the difficulty of assigning a monetary value to health outcomes. As such, we tend to use cost-effectiveness analysis, which does not require outcomes to be valued monetarily.

Economists and decision-makers are often interested in comparing outcomes in terms of individuals' or society's preferences over different health outcomes (National Institute for Health and Clinical Excellence, 2008). This is because the value that an individual attaches to a health state is important; a long distance runner may prefer a broken arm to a broken toe, while the opposite may be true for a swimmer. This dynamic can be captured in a cost-effectiveness analysis by measuring the effect of an intervention on a person's health in terms of its impact on their 'utility'. When preferences are considered in this way, the analysis is often referred to as a cost-utility analysis, which is what we implement in this study.

\section{Costs}

Economic evaluations require information on resource use, to which cost figures or estimates can be attached. In an economic evaluation in healthcare, the primary cost of an intervention tends to be the direct medical costs. These will include the costs of such resource use as staff time, drugs and equipment. Evaluations can also account for wider societal costs, such as patients' travel costs, out of pocket expenditure or productivity losses. When estimating the cost of an intervention it is important to adopt an appropriate 'perspective'; usually that of the health service or of society more broadly.

For the HOVIS study, information on resource use was collected in the trial by the staff completing home visits and interviews. Our analysis adopts a National Health Service (NHS) perspective; considering the cost of home visits for the NHS. Resource use included staff attending time, travel time, travel cost, administration time and equipment. Information on staff NHS pay bands was recorded in the trial. Unit costs were then attached to resource use, using NHS staff earnings estimates (Department of Health, 2011) and the Unit Costs of Health \& Social Care 2011 (Curtis, 2011). Staff costs were the primary cost driver in this occupational therapy intervention and depended on NHS pay bands, including oncosts (e.g. overheads), as shown in Table 1. Hospital policies 
generally required that two members of staff be in attendance. In some instances a student was present for educational purposes.

[Table 1 about here]

\section{Outcomes}

In a cost-effectiveness analysis, the effectiveness of an intervention can be estimated using the outcome measures commonly used in the appropriate clinical area. In stroke rehabilitation this might include a scale measure such as the Barthel Index, as collected and reported in the HOVIS study (Drummond et al., 2013), or might be a count of a relevant outcome such as number of falls or mortality. However, measures such as these are not relevant to all clinical fields and all healthcare interventions, and decision-makers must make funding decisions across different clinical areas. As such it is necessary to compare stroke rehabilitation interventions with interventions for other physical and mental health problems. In order to address this problem measures have been developed that capture the extent to which a given health problem or intervention affects an individual's overall well-being or 'utility'. A common outcome measure, advised by the NICE reference case (National Institute for Health and Clinical Excellence, 2008), is the quality-adjusted life year (QALY). QALYs combine information about length and quality of life to produce a generic measure of health outcome. The quality of life part is defined by a measure of healthrelated quality of life (HRQOL). An individual's HRQOL is anchored on a 0 to 1 scale, such that 1 represents 'full health' and 0 represents being dead (or in an equivalent health state). The value of a person's HRQOL is then multiplied by the number of years in this health state to produce the number of QALYs (Drummond et al., 2005). When a cost-effectiveness analysis is carried out using QALYs it is often referred to as a cost-utility analysis. This is the form taken by our analysis.

Outcomes were collected from participants in the HOVIS study. The primary outcome of interest for the economic evaluation was the EQ-5D (EuroQol Group, 1990). The EQ-5D is a generic measure of HRQOL. It includes 5 questions or 'dimensions': mobility, self-care, usual activities, pain/discomfort and anxiety/depression. Each question has 3 possible responses or levels: no problems, some problems and major problems. The EQ-5D is therefore able to define 243 different health states; 245 including dead and unconscious. Other similar measures are available, including the SF-6D (Brazier et al., 2002) and Health Utilities Index (Torrance et al., 1982). The EQ-5D is the preferred instrument of NICE (National Institute for Health and Clinical Excellence, 2008) and has previously been found to be responsive in patients with a stroke (Pickard et al., 2005). The importance of each dimension of health for an individual's healthrelated quality of life is estimated based on the desirability of a particular health state, meaning that it is necessary to attach value weights to EQ-5D responses. These weights are obtained by using public valuation exercises and regression analyses, which have been carried out in a number of countries including the UK (Szende et al., 2007). These valuations are anchored such that 1 represents 
'full health' and 0 respresents being dead. Once valued in this way, EQ-5D data are used to estimate QALYs, as described above.

\section{Cost-effectiveness}

The cost-effectiveness of an intervention is presented in the form of an incremental cost-effectiveness ratio (ICER), which represents the cost-per-benefit of an intervention. An ICER is calculated by dividing the incremental cost of an intervention by the incremental benefit of the intervention:

$$
I C E R=\frac{\left(C_{1}-C_{0}\right)}{\left(B_{1}-B_{0}\right)}
$$

where $C_{1}$ and $B_{1}$ are the cost and benefit of the intervention being evaluated and $C_{0}$ and $B_{0}$ are the cost and benefit of the control. In the case of our analysis $C_{1}$ and $B_{1}$ are the costs and benefits of home visits, and $C_{0}$ and $B_{0}$ are the costs and benefits of hospital-based interviews.

As with all quantitative analyses, it is necessary to take into consideration the uncertainty in our estimates. An ICER is a ratio value, which is not observed at the individual level. As such it is not appropriate to consider p-values in the usual way as we cannot observe uncertainty around the ICER. A solution is to resample using the individual-level cost and outcome data using a method called bootstrapping (Briggs et al., 1997). Bootstrapping does not require assumptions about the distribution of costs and outcomes and is therefore useful when we do not know these. Using this method we are able to produce a cost-effectiveness plane, which shows simulated combinations of mean costs and outcomes and the nature of the uncertainty in our estimates.

Judgements about whether an intervention is cost-effective depend on whether the incremental benefit is worth the incremental cost; that is, how much decisionmakers are willing to pay for a single unit of the health outcome. In the case of QALYs, NICE adopts a willingness-to-pay of $£ 20,000-£ 30,000$ per QALY. This value can be incorporated into an economic evaluation using the net benefit approach (Claxton, 1999). The net benefit approach works by multiplying the incremental outcome by the willingness-to-pay value, and subtracting the incremental cost from this:

$$
N B=\lambda\left(B_{1}-B_{0}\right)-\left(C_{1}-C_{0}\right)
$$

where $\lambda$ is the willingness-to-pay for a single unit of the measure of benefit ' $B$ '. It is also useful to present decision-makers with some information regarding the uncertainty surrounding our cost-effectiveness estimates. As described above, we employ methods of bootstrapping to capture the uncertainty of our estimates. These can then be used to estimate the probability that an intervention is cost-effective at a given threshold cost per QALY. Generally in economic evaluation, statistical inference is ignored and the intervention that maximises mean net benefit is judged to be the optimal choice (Claxton, 1999). 


\section{Results}

The main results of the HOVIS trial are reported elsewhere (Drummond et al., 2013). For the economic evaluation, cost and outcome data were available for 28 people in the control group and 37 people in the treatment group.

\section{Costs}

Resource use collected in the trial is shown in Table 2. The primary resource use relating to home visits and interviews was that of staff time. Hospital interviews involved either 1 or 2 members of staff, while home visits included up to 3 . In all cases the primary member of staff tended to be a band 6 but, for all staff involved, bands ranged from 2 to 7 . For home visits there was an additional cost of travel time and taxi fares, which were recorded in the trial.

Total costs are also shown in Table 2. For the whole sample, the total cost of a home visit, on average, was $£ 183$, compared with $£ 75$ for hospitalbased interviews. The incremental cost of home visits is therefore $£ 108$. When individuals without complete outcome data are excluded, as in the economic evaluation, the incremental cost is $£ 115$.

[Table 2 about here]

\section{Outcomes}

From baseline to follow-up, mean EQ-5D values increased from 0.450 to 0.512 in the control arm, compared with 0.406 to 0.527 in the intervention arm. The incremental effect of home visits at one month, in terms of EQ-5D score, was therefore 0.065. Assuming that this benefit lasts for the 1 month following discharge from hospital, and no longer, this gives an incremental QALY benefit of 0.005 .

\section{Cost-effectiveness}

Utility-weighted EQ-5D data were combined with cost data to produce a costeffectiveness analysis. The ICER, or cost-per-QALY of home visits, was £21,987. Table 3 shows the results for individuals with full baseline and follow-up outcome data. As discussed above, it is important to take into account the uncertainty around these estimates. Figure 1 shows a cost-effectiveness plane based on 1000 bootstrapped simulations. From this graph we can see that, while the majority of points are in the upper-right quadrant (indicating higher cost and higher effectiveness), many are in the upper left quadrant (indicating higher cost and lower effectiveness). $76 \%$ of estimates lie in the upper-right quadrant.

We can also estimate the probability that the intervention will be costeffective at a given willingness-to-pay value. Plotted on Figure 1 is a line indicating a willingness-to-pay value of $£ 20,000$ per QALY, as used by NICE. This line is plotted from the origin to the point where incremental cost is $£ 20,000$

and incremental benefit is 1 QALY. All points below this line indicate that the 
intervention is cost-effective at a willingness-to-pay of $£ 20,000$ per QALY. All points above this line indicate that home visits are not more cost-effective than hospital interviews. Based on this analysis of a small sample, we estimate that there is a $47 \%$ chance that home visits are cost-effective at a willingness-to-pay of $£ 20,000$ per QALY.

[Figure 1 about here] [Table 3 about here]

\section{Discussion and implications}

Our observed results suggest that home visits may be cost-effective at a willingnessto-pay of $£ 20,000$ - $£ 30,000$ per QALY. However, due to the small scale of this study and the amount of missing data, these results should be considered with caution. Our results demonstrate a high level of uncertainty in our data due to a small sample and large variability in costs and outcomes. Based on our analysis there is a reasonably high probability (24\%) that home visits are more expensive and less effective than hospital interviews. Furthermore, due to missing data, it is likely that we substantially underestimated the cost of home visits. For example, in many cases no 'additional time' was reported for home visits. This should include the time spent writing-up, and as such a result of no additional time is unrealistic. Missing data were mainly due to incomplete staff time recording forms. For our outcome measure, EQ-5D, data were only missing in 2 cases.

Our results consider the consequences of home visits in terms of healthrelated quality of life only. An economic evaluation could account for other important outcomes such as functioning or mobility. Home visits may be considered to facilitate a better planned discharge, preventing the need for readmissions to hospital and greater input from community services. Clearly there are other important costs to consider that were not included in our study. In future studies it will be important to include data on the use of health and social services, medication, and whether or not individuals return to work. Collection of such data can be facilitated by the use of validated generic and condition-specific instruments, of which a database is available (Ridyard and Hughes, 2012).

These results were obtained as part of a feasibility randomised controlled trial which may not necessarily be representative of clinical practice. The visits in this study were shared between a research occupational therapist and occupational therapists from the stroke unit where the study was carried out. These results only include the patients who were randomised in the study and exclude the cohort of patients for whom a home visit was considered to be essential. We compared a home visit to a hospital-based interview (control group), which is not currently standard practice.

Our analysis represents the first economic evaluation of pre-discharge occupational therapy home visits after stroke. There are no previously published findings with which to compare our results, though other studies are underway (Wales et al., 2012). We hope our study will support future economic evaluations 
of occupational therapy interventions, including pre-discharge home visits.

\section{Conclusion}

Our results are based on data collected in the biggest trial of occupational therapy pre-discharge home visits to date, and this is therefore the most comprehensive economic evaluation of this intervention. However, it would be unreasonable to rely on data of this nature for policy decisions. The only conclusion one can comfortably make based on these results is that a larger trial of occupational therapy home visits, with more comprehensive data collection and a stricter adherence to the research protocol, is warranted. 


\section{Key findings}

- The average cost of a home visit was $£ 183$, compared with $£ 75$ for a hospital interview

- The cost-effectiveness of home visits is extremely uncertain

- Wider use of economic evaluations is necessary in occupational therapy

\section{What the study has added}

This is the first study to examine the cost-effectiveness of occupational therapy pre-discharge home visits after stroke. Results suggest that home visits may be cost-effective.

\section{Acknowledgements}

We are grateful to Karen Fellows, Dr Nikola Sprigg, Prof Nadina Lincoln, the other members of the HOVIS research team, steering committee and expert advisors; Dr Nicola Brain, Claire Edwards, Dr Boliang Guo, Dr Annie McCluskey, Mr Oswald Newell, Dr Cecily Palmer, Dr Ruth Parry, Dr Kate Radford, Dr Tracy Sach, Professor Cath Sackley, Dr Karen Stainer and Professor Marion Walker.

We would also like to thank the staff from the stroke rehabilitation unit at the Royal Derby Hospital, and particularly the occupational therapists who were involved in the HOVIS study.

This paper presents independent research commissioned by the National Institute for Health Research (NIHR) as part of the Collaboration for Leadership in Applied Health Research and Care - Nottinghamshire, Derbyshire and Lincolnshire (CLAHRC-NDL). The views expressed are those of the authors and not necessarily those of the NHS, the NIHR or the Department of Health. 


\section{References}

Barras, S. (2005). A systematic and critical review of the literature: The effectiveness of Occupational Therapy Home Assessment on a range of outcome measures. Australian Occupational Therapy Journal, 52(4):326-336.

Brazier, J., Roberts, J., and Deverill, M. (2002). The estimation of a preferencebased measure of health from the SF-36. Journal of Health Economics, 21(2):271-292.

Briggs, A. H., Wonderling, D. E., and Mooney, C. Z. (1997). Pulling costeffectiveness analysis up by its bootstraps: A non-parametric approach to confidence interval estimation. Health Economics, 6(4):327 - 340.

Claxton, K. (1999). The irrelevance of inference: a decision-making approach to the stochastic evaluation of health care technologies. Journal of health economics, 18(3):341-364.

College of Occupational Therapists (2007). Building the Evidence for Occupational Therapy: Priorities for Research. College of Occupational Therapists, London.

Curtis, L. (2011). Unit Costs of Health and Social Care 2011. Personal Social Services Research Unit, Canterbury.

Department of Health (2011). NHS Staff Earnings Estimates, July to September 2011. Technical report, Information Centre for Health and Social Care.

Drummond, A. E. R., Whitehead, P., Fellows, K., Edwards, C., and Sprigg, N. (2012). Occupational therapy predischarge home visits for patients with a stroke: what is national practice? The British Journal of Occupational Therapy, 75(9):396-402.

Drummond, A. E. R., Whitehead, P., Fellows, K., Sprigg, N., Sampson, C. J., Edwards, C., and Lincoln, N. (2013). Occupational therapy predischarge home visits for patients with a stroke (HOVIS): results of a feasibility randomized controlled trial. Clinical rehabilitation, 27(5):387-97.

Drummond, M. F., Sculpher, M. J., Torrance, G. W., O'Brien, B. J., and Stoddart, G. L. (2005). Methods for the Economic Evaluation of Health Care Programmes. Oxford University Press.

EuroQol Group (1990). EuroQol - a new facility for the measurement of healthrelated quality of life. Health policy, 16(3):199-208.

Hibberd, J. (2008). The home-visiting process for older people in the in-patient intermediate care services. Quality in Ageing and Older Adults, 9(1):13-23.

Johnston, K., Barras, S., and Grimmer-Somers, K. (2010). Relationship between pre-discharge occupational therapy home assessment and prevalence of postdischarge falls. Journal of evaluation in clinical practice, 16(6):1333-9. 
Lannin, N. A., Clemson, L., and McCluskey, A. (2011). Survey of current pre-discharge home visiting practices of occupational therapists. Australian occupational therapy journal, 58(3):172-7.

Lannin, N. A., Clemson, L., McCluskey, A., Lin, C.-W. C., Cameron, I. D., and Barras, S. (2007). Feasibility and results of a randomised pilot-study of predischarge occupational therapy home visits. BMC health services research, $7: 42$.

Logan, P. A., Elliott, R. A., Quinn, C., Gladman, J. R. F., and Walker, M. F. (2011). Final Report: A study of long term economic consequences of short term stroke occupational therapy. Technical report, University of Nottingham.

National Institute for Health and Clinical Excellence (2008). Guide to the methods of technology appraisal. Technical report, National Institute for Health and Clinical Excellence, London.

Patterson, C. J. and Mulley, G. P. (1999). The effectiveness of predischarge home assessment visits: a systematic review. Clinical Rehabilitation, 13(2):101-104.

Patterson, C. J., Viner, J., Saville, C., and Mulley, G. P. (2001). Too many predischarge home assessment visits for older patients? A postal questionnaire survey. Clinical Rehabilitation, 15(3):291-295.

Pickard, A. S., Johnson, J. A., and Feeny, D. H. (2005). Responsiveness of generic health-related quality of life measures in stroke. Quality of Life Research, 14(1):207-19.

Ridyard, C. H. and Hughes, D. A. (2012). Development of a database of instruments for resource-use measurement: purpose, feasibility, and design. Value in Health, 15(5):650-5.

Szende, A., Oppe, M., Devlin, N. J., and EuroQol Group (2007). EQ-5D Value Sets, volume 2 of EuroQol Group Monographs. Springer Netherlands, Dordrecht.

Torrance, G. W., Boyle, M. H., and Horwood, S. P. (1982). Application of multi-attribute utility theory to measure social preferences for health states. Operations research, 30(6):1043-69.

Wales, K., Clemson, L., Lannin, N. A., Cameron, I. D., Salked, G., Gitlin, L., Rubenstein, L., Barras, S., Mackenzie, L., and Davies, C. (2012). Occupational therapy discharge planning for older adults: a protocol for a randomised trial and economic evaluation. BMC geriatrics, 12(1):34.

Welch, A. and Lowes, S. (2005). Home assessment visits within the acute setting: a discussion and literature review. The British Journal of Occupational Therapy, 68(4):158-164. 


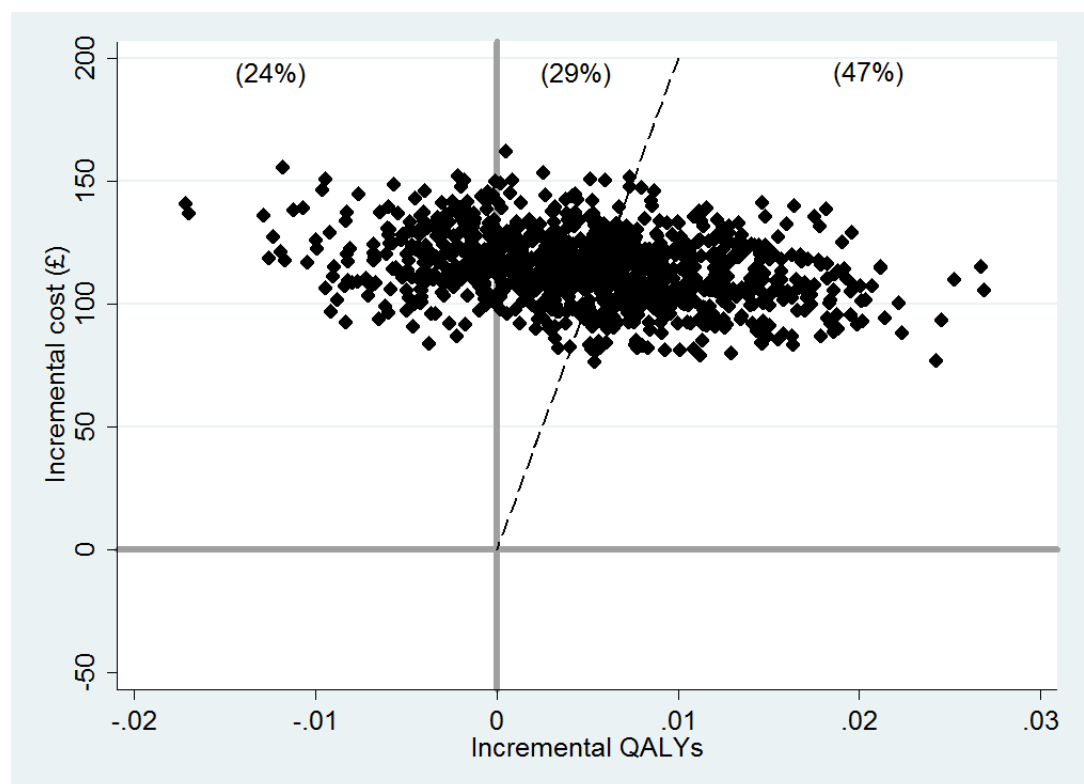

Figure 1: Cost-effectiveness plane

\begin{tabular}{c|c|c} 
& Per annum & Per hour \\
\hline Band 2 & $£ 19,031.28$ & $£ 12.29$ \\
Band 3 & $£ 21,715.08$ & $£ 14.02$ \\
Band 4 & $£ 25,549.08$ & $£ 16.50$ \\
Band 5 & $£ 28,744.08$ & $£ 18.56$ \\
Band 6 & $£ 37,562.28$ & $£ 24.25$ \\
Band 7 & $£ 47,275.08$ & $£ 30.52$
\end{tabular}

Table 1: NHS staff pay bands

\begin{tabular}{l|c|c|c|c|c|c} 
& \multicolumn{3}{|c|}{ Home visits } & \multicolumn{3}{c}{ Interview } \\
\hline Staff time (mins) & Obs & Mean & s.d. & Obs & Mean & s.d. \\
\hline Organising & 39 & 29 & 25 & - & - & - \\
Completing & 40 & 73 & 26 & 30 & 76 & 29 \\
Travelling & 38 & 31 & 19 & - & - & - \\
Additional & 27 & 86 & 52 & 10 & 69 & 27 \\
(of which report writing) & 20 & 92 & 40 & 10 & 61 & 13 \\
\hline Staff band & Obs & Median & & Obs & Median & \\
\hline Staff A & 42 & 6 & & 41 & 6 & \\
Staff B & 38 & 3 & & 11 & 5 & \\
\hline Costs & Obs & Mean & s.d. & Obs & Mean & s.d. \\
\hline Travel costs & 41 & $£ 26$ & 16 & - & - & - \\
Total costs & 42 & $£ 183$ & 81 & 30 & $£ 75$ & 40 \\
\hline
\end{tabular}

Table 2: Resource use by occupational therapists on pre-discharge interventions 


\begin{tabular}{|c|c|c|}
\hline & Observed mean & BS $95 \%$ confidence interval * \\
\hline $\begin{array}{l}\text { Home visits } \\
\text { Costs } \\
\text { QALYs }\end{array}$ & $\begin{array}{c}£ 187.71 \\
0.010\end{array}$ & $\begin{array}{c}£ 164.82-£ 216.00 \\
0.001-0.019\end{array}$ \\
\hline $\begin{array}{l}\text { Interview } \\
\text { Costs } \\
\text { QALYs }\end{array}$ & $\begin{array}{l}£ 73.03 \\
0.005\end{array}$ & $\begin{array}{c}£ 58.23-£ 86.81 \\
-0.005-0.015\end{array}$ \\
\hline $\begin{array}{l}\text { Incremental } \\
\text { Costs } \\
\text { QALYs } \\
\text { ICER }\end{array}$ & $\begin{array}{c}£ 114.68 \\
0.005 \\
£ 21,986.92\end{array}$ & $\begin{array}{c}£ 113.31-£ 115.09 \\
0.005-0.006 \\
-£ 34,468-£ 12,551.31\end{array}$ \\
\hline
\end{tabular}

Table 3: Observed and bootstrapped (BS) estimates of incremental costs and QALYS 